\title{
Kleptomanie: Überblick zum Forschungsstand und Ergebnisse einer verhaltenstherapeutischen Studie
}

\author{
G. Sauke \\ Verhaltenstherapie-Ambulanz, Universitätsklinikum Eppendorf, Hamburg, Deutschland
}

\author{
Schlüsselwörter \\ Kleptomanie: Forschungsstand, "ursachenorientierte» \\ Verhaltenstherapie, Therapieerfolg
}

\section{Zusammenfassung}

Hintergrund: Kleptomanie ist mit "Mythen» behaftet. Es mangelt an systematischen Untersuchungen; vorliegende Behandlungsstudien beinhalten kleine Fallzahlen (1-20) und sind nicht kontrolliert. Nicht von ungefähr meldete "Der Spiegel online" am 10. November 2002: "US-Forscher fahnden nach Kleptomanen" für eine medikamentöse Studie. Verhaltenstherapeuten haben bisher überwiegend Einzelfallstudien vorgelegt, in denen mehrheitlich «klassisch» symptomzentriert vorgegangen wurde. Nach ausführlicher Darstellung des aktuellen Forschungsstandes wird eine eigene Untersuchung dargestellt, die sich an einem Modell zur Behandlung pathologischer Spieler orientiert. Ziel: Es sollte eine individuelle, "ursachenorientierte» Verhaltenstherapie bei Betroffenen mit Kleptomanie (nach DSM-III-R) durchgeführt werden und anhand von drei Beurteilungskriterien ausgewertet werden. Patienten und Methode: 12 Patienten beendeten die Therapie und konnten bis zum Abschluss der Studie nachuntersucht werden. Eingebettet in eine Verhaltens-, Bedingungs- und Funktionsanalyse erfolgten "ursachenorientierte» Interventionen auf der Ebene der herausgearbeiteten auslösenden und aufrechterhaltenden Bedingungen und Funktionen des kleptomanischen Verhaltens. Ergebnisse: 9 Patienten erfüllten alle Beurteilungskriterien für Therapieerfolg. Schlussfolgerungen: Die Ergebnisse sind ermutigend. Replikationsuntersuchungen mit größerer Fallzahl sind erforderlich, um die Ergebnisse zu stützen oder zu verwerfen.

\section{Key Words}

Kleptomania: state of research, 'cause-orientated' behavior therapy, therapy outcome

\section{Summary}

Kleptomania: Overview of the State of Research and Results of a Behavior Therapy Study

Kleptomania is surrounded with 'myths'. There is a shortage of systematic research. Treatment studies comprise few cases (1-20) and are not controlled. It is not without reason that 'Der Spiegel online' reported on November 10, 2002: 'U.S. scientists are searching for kleptomaniacs' for clinical studies'. To date, behavior therapists have mainly supplied individual case studies. In the majority of these studies, 'classical' symptom-centered therapy was employed. After detailed illustration of the current state of research, the author presents her own study based on a treatment model for pathological gamblers. Goal: To carry out an individual 'cause-orientated' behavior therapy for patients suffering from kleptomania (according to DSM-III-R), and to evaluate this treatment according to three assessment criteria. Patients and Method: 12 patients completed the therapy and could subsequently be assessed until completion of the study. Embedded in behavior, condition and function analysis, interventions took place at the level of those variables regarded as 'causal' factors in triggering and maintaining the kleptomaniac behavior. Results: 9 patients met all assessment criteria for success. Conclusions: The results are encouraging. Replication studies with an increased number of cases are required to support or dismiss these findings.

\begin{tabular}{ll}
\hline KARGER & @ 2004 S. Karger GmbH, Freiburg \\
Fax +497614520714 & Accessible online at: \\
$\begin{array}{l}\text { E-mail Information@Karger.de } \\
\text { www.karger.com }\end{array}$ & www.karger.com/ver
\end{tabular}




\section{Überblick zum aktuellen Forschungsstand}

Zum Thema Kleptomanie mangelt es an systematischen Untersuchungen. Fallberichte zur Kleptomanie gibt es seit den Anfängen des 19. Jahrhunderts aus verschiedenen psychiatrischen und psychologischen Denkrichtungen und Zeitströmungen sowie aus verschiedenen Ländern aus der ganzen Welt. So ist eine reichhaltige Literatur entstanden.

McElroy et al. [1991a] sichteten Fallberichte oder kleine Fallserien von psychiatrischen Patienten mit Kleptomanie (1953-1989; 28 Autoren), Studien über verhaftete Ladendiebe und Begutachtungsstudien und Studien über Kleptomanie bei Patienten mit Essstörungen.

Goldman [1991] kritisiert aufgrund einer Literaturrecherche (1968-1989; 22 Autoren) insbesondere, dass es an ausführlicher Informationen mangelt, die Autoren meist nur einen oder zwei Aspekte eines Falles darstellen, die Veröffentlichungen eher auf Kurzinterviews beruhen und viele Fälle möglicherweise wegen «interessanter Eigenschaften» (wie z.B. sexuelle Dysfunktionen) zur Publikation ausgewählt wurden. Nach operationalisierten diagnostischen Kriterien ist Kleptomanie bisher selten und mit höchstens 37 Fällen untersucht worden. Forschungshindernisse liegen möglicherweise in der Verheimlichungstendenz bei dieser Störung. Verschiedentlich heißt es, dass Betroffene erst unter dem Druck härterer und als sozial besonders bedrohlich empfundener Strafen Hilfe suchen [Möller, 1977] oder wegen anderer psychiatrischer Störungen in Behandlung kommen und das Stehlen vor ihren Therapeuten und ihren Familien aus Scham verheimlichen [McElroy et al., 1995; Wiedemann, 1998].

\section{Historische und klinische Beschreibung}

Der Terminus Kleptomanie (gr. kleptein: stehlen; mania: Raserei, Wahnsinn, Besessenheit) etablierte sich mit Marc [1840, zit. n. Müllener, 1964] in der Wissenschaftsgeschichte. Als nosologischer Begriff löste sich Klopemanie [Matthey, 1816, zit. n. Müllener, 1964] (gr. klopeia: Diebstahl), später Kleptomanie, aus der Typisierung einer «Manie ohne Delirium» [Pinel, 1801] und deren Differenzierung in der Lehre von den Monomanien [Esquirol, 1838].

Die hier interessierende «instinktive Monomanie» beschrieb Esquirol als eine «Willensverletzung», bei der der Kranke zu Handlungen hingezogen werde, «zu denen ihn weder Vernunft, noch Gefühl bestimmen, und welche sein Gewissen missbilligt. Aber er hat nicht die Kraft, sie zu unterdrücken; die Handlungen geschehen unfreiwillig, instinctartig» [Bernhard, 1838, S. 1f.].

Marc definierte Kleptomanie als «a conscious urge to steal occurring in an individual in whom there is no ordinary disturbance of consciousness. The individual concerned frequently strives against this urge, but by its nature it is irresistible» [zit. n. Aggernaes, 1961, S. 1]. Auch Kraepelin, der den «Stehl- trieb» (die Kleptomanie) vor der Formulierung des «impulsiven Irreseins» führte, sah «die Krankhaftigkeit dieser Handlungen darin, dass denselben jeder verständige Beweggrund fehlt» [1904, S. 796].

Nachdem diese Krankheitskonzepte verlassen worden waren, hielt sich der Begriff der Kleptomanie unter verschiedenen ätiopathogenetischen Betrachtungen in der Literatur [Cierpka, 1986], wobei je nach Autor und wissenschaftlicher Herkunft vom «Stehlen um des Stehlens und nicht um des Diebesgutes willen» [Hirschmann, 1955], von «unwiderstehlicher Stehlsucht», «Stehldrang», «Kleptomanie» (soviel wie Stehltrieb oder Stehlzwang), «Stehltrieb» (im Sinne von Antrieb, Drang oder Sucht) [von Schumann, 1988] oder im psychoanalytischen Verständnis auch von «symbolischen Diebstählen» gesprochen wird.

Nach Cierpka bezeichnet Kleptomanie «im engeren Sinne «pathologisches Stehlen〉 im Zustand der Erregung und Anspannung ..., also die dranghafte Entladungstendenz einer inneren Unruhe» [1986, S. 95].

Nach von Schumann gehört zum Wesen der Kleptomanie «die Entladungstendenz eines psychosomatischen Antriebes, einer unlustvollen Unruhe und explosiblen Spannung, die nur bei manchem, also keineswegs bei allen, sexuell getönt oder sogar sexuell akzentuiert ist ... Entscheidend bei der Kleptomanie ist der Stehlakt als solcher, nicht der Besitz des Gestohlenen; die Zueignung ist ein nicht beabsichtigter Nebeneffekt» [1988, S. 13]. McElroy et al. [1991a] verweisen bei Kleptomanie auf impulsive und kompulsive Kennzeichen. Kleptomanisches Stehlen erfolge abrupt, plötzlich, unvorhergesehen, aus einem Impuls, Drang, Antrieb heraus, ohne Vorbedacht, Vorausplanung, Zusammenarbeit mit anderen, ohne volle Berücksichtigung der Möglichkeit einer Festnahme. Kleptomanie ähnele auch einem Zwang. Es sei ein wiederholtes Verhalten, das ausgeführt werde, um Unbehagen zu neutralisieren; es sei verbunden mit einem Dringlichkeitsgefühl und Angstanstieg, wenn dem Stehlimpuls Widerstand geleistet werde, und mit Spannungsreduktion oder Erleichterung beim Nachgeben und Ausführen der Handlung. Die Ausführung der Handlung werde von den Betroffenen als unvernünftig oder falsch erkannt. Anders als «gewöhnliche» Diebe erlebten Menschen mit Kleptomanie ihr Verhalten konflikthaft. Sie fühlten sich gezwungen oder getrieben zu stehlen. Versuche, sich zu widersetzen oder gegen den Antrieb anzukämpfen, seien oft erfolglos, «because the drive seems irresistible or beyond voluntary control» [1991, S. 94].

\section{Diagnostische Merkmale und Differentialdiagnose}

Die erstmals 1980 gegebene operationalisierte Definition von Kleptomanie [DSM-III, APA] findet sich fast unverändert in den Folgemanualen und ist mit der Definition der WHO [ICD-10, 1991] eng verwandt. Die diagnostischen Kriterien für Kleptomanie (DSM-IV 312.32, ICD-10 F 63.2) sind: 
A Wiederholtes Versagen, Impulsen zum Stehlen von Gegenständen zu widerstehen, die weder zum persönlichen $\mathrm{Ge}$ brauch noch wegen ihres Geldwertes benötigt werden.

B Zunehmendes Gefühl von Spannung unmittelbar vor Begehen des Diebstahls.

C Vergnügen, Befriedigung oder Entspannung beim Begehen des Diebstahls.

D Das Stehlen wird nicht begangen, um Wut oder Rache auszudrücken, und erfolgt nicht als Reaktion auf Wahnphänomene oder Halluzinationen.

E Das Stehlen kann nicht besser durch eine Störung des Sozialverhaltens, eine manische Episode oder eine antisoziale Persönlichkeit erklärt werden.

Als weitere diagnostische Merkmale gelten, dass die Gegenstände gestohlen werden, obwohl sie typischerweise von geringem Wert für den Betroffenen sind, der es sich leisten könnte, sie zu bezahlen, der sie oft verschenkt, wegwirft, sammelt oder heimlich zurückgibt. Personen mit dieser Störung vermeiden üblicherweise das Stehlen, wenn eine unmittelbare Festnahme befürchtet werden muss (z.B. vor den Augen eines Polizisten). Das Stehlen ist gewöhnlich nicht vorausgeplant, und das Risiko der Festnahme wird nicht in vollem Umfang berücksichtigt. Das Stehlen geschieht ohne Hilfe oder Mitwirkung anderer.

Nach DSM-III-R/DSM-IV [APA, 1987, 1994] ist Kleptomanie zu unterscheiden von:

- Gewöhnlichen Diebstahlsvergehen oder Ladendiebstahl. (Gewöhnlicher Diebstahl ist geplant oder impulsiv, wird absichtlich begangen und ist durch den Gebrauchs- oder Geldwert des gestohlenen Gegenstandes motiviert.)

- Simulation der Symptome der Kleptomanie, um einer Strafverfolgung zu entgehen.

- Antisozialer Persönlichkeitsstörung und Störung des Sozialverhaltens. (Diese unterscheiden sich von Kleptomanie durch ein allgemeines Muster antisozialen Verhaltens.)

- Von beabsichtigtem oder fahrlässigem Stehlen, das während einer manischen Episode, als Reaktion auf Wahnphänomene oder Halluzinationen (z.B. bei Schizophrenie) oder im Rahmen einer Demenz auftreten kann.

\section{Diagnostische Zuordnung}

Im DSM-IV fällt Kleptomanie in die diagnostische Kategorie der «Störungen der Impulskontrolle», die nicht an anderer Stelle klassifiziert sind. Zu dieser Kategorie gehören auch die intermittierende explosible Störung, das pathologische Spielen, Pyromanie, Trichotillomanie und Störungen der Impulskontrolle, nicht näher bezeichnet. Im ICD-10 [Dilling et al., 1991; DIMDI, 2004] erscheint pathologisches Stehlen (Kleptomanie) unter «Abnorme Gewohnheiten und Störungen der Impulskontrolle». Für die Zusammenstellung dieser Störungen unklarer Ursache werden gewisse Ähnlichkeiten in der Beschreibung geltend gemacht, vor allem wiederholte Handlungen ohne vernünftige Motivation; Betroffene berichten von Impulsen, die sie nicht kontrollieren können [Rösler, 1993].
Andere Autoren verstehen Kleptomanie als eine Form der «behavioural (non-chemical) addictions» [Marks, 1990] oder als eine Form der «behavioral dependence» [Lejoyeux et al., 2000]. McElroy et al. [1995] schlagen vor, Kleptomanie, zwanghaftes Kaufen und Binge Eating als «compulsive-impulsive spectrum disorders» zu klassifizieren und den «affective spectrum disorders» [Hudson und Pope, 1990] zuzuordnen.

Nach Tynes et al. [1990] ist Kleptomanie eine mögliche Variante der Zwangsstörung. Kleptomanie weise auf phänomenologischer Ebene Gemeinsamkeiten zur Zwangsstörung auf, aber auch Unterschiede [Presta et al., 2002]. Ersten Forschungsergebnissen zufolge leiden Angehörige ersten Grades ähnlich häufig an Zwängen und/oder affektiven Störungen. Nach Presta et al. [ebd.] empfiehlt sich die Zuordnung der Kleptomanie zu den «obsessive-compulsive spectrum disorders» [Hollander und Wong, 1995] auf einem Kontinuum zwischen Impulsivität und Kompulsivität. Die in diesem Spektrum aufgeführten Störungen sind durch sich aufdrängende Zwangsgedanken und/oder sich wiederholende zwanghafte Verhaltensweisen gekennzeichnet und weisen hinsichtlich Ätiologie, familiärer und demographischer Merkmale, Neurobiologie, Komorbidität, klinischem Verlauf und Reaktion auf zwangsspezifische Verhaltenstherapie und Pharmakotherapie viele Gemeinsamkeiten zur Zwangsstörung auf [Neudecker und Hand, 1999].

\section{Forschungsergebnisse}

\section{Epidemiologische Daten}

Die Häufigkeit von Kleptomanie ist nicht genau bekannt. Bevölkerungsstudien liegen nicht vor. Nach Goldman [1998] liegt die Prävalenz der Kleptomanie bei wenigstens 0,6\%. Sie betrifft Menschen aus allen sozialen Schichten [von Schumann, 1969; Goldmann, 1998]. Unter Zugrundelegung operationalisierter Kriterien dürfte Kleptomanie bei weniger als $5 \%$ der verhafteten Ladendiebe vorliegen [DSM-IV-TR, APA, 2000]. Die Definitionskriterien sind eng gefasst, es existieren subsyndromale Varianten, bei denen nicht alle DSMKriterien zutreffen [McElroy et al., 1991a, 1995; Sarasalo et al., 1997]. Nach Lejoyeux et al. [2000] variiert die in Studien ausgewiesene Kleptomanie-Rate unter verhafteten Ladendieben zwischen 0 und $24 \%$.

In klinischen Stichproben sind nach bisherigen Befunden etwa zwei Drittel der Kleptomaniepatienten weiblich [Goldman, 1998; DSM-IV-TR, APA, 2000]. In geschlechtsgemischten Studien erstreckt sich die Altersspanne auf 17-71 Jahre [McElroy et al., 1991a, b; Goldman, 1991; Sarasalo et al., 1996; Wiedemann, 1998; Presta et al., 2002] mit einem leichten Überwiegen der Fälle im zweiten und dritten Lebensjahrzehnt [McElroy et al., 1991a]. Je nach Studie waren 35-62\% der Betroffenen verheiratet, $25-65 \%$ niemals verheiratet, $4-17 \%$ geschieden, $0-8 \%$ verwitwet, $35-70 \%$ berufstätig, $30-65 \%$ ohne eigenes Einkommen (in Ausbildung, arbeitslos, Hausfrau, 
Rentner). In der Berufsgruppenverteilung ließen sich 5-42\% dem White-Collar-, 25-35\% dem Blue-Collar-Arbeitsbereich zurechnen. Bei Presta et al. verfügten $75 \%$ über eine höhere Schulbildung.

\section{Beginn, Verlauf, andere Merkmale}

Die Störung kann in der Kindheit, der Adoleszenz, im Erwachsenenalter und selten auch im späten Erwachsenenalter (>65 Jahre) beginnen [Kossak, 1985; McNeilly und Burke, 1998]. Goldman [1991] fand bei Frauen ein durchschnittliches Erstmanifestationsalter von 20 Jahren (Range 6-44); in geschlechtsgemischten Stichproben von Wiedemann [1998] lag die Erstmanifestation bei 30 Jahren (Range 16-54), bei Presta et al. [2002] bei 20 Jahren (Range 12-28). Obwohl nur wenig systematische Informationen vorliegen, werden drei typische Verläufe beschrieben [DSM-IV-TR, APA, 2000]:

- sporadisch mit kurzen Episoden und langen Zeiten der Remission;

- episodisch mit lang andauernden Perioden des Stehlens und Perioden der Remission;

- chronisch mit einem gewissen Maß an Fluktuation.

Die Störung kann trotz wiederholter Verurteilungen wegen Ladendiebstahls jahrelang fortdauern [ebd.]. Bei McElroy et al. [1991b] lag die durchschnittliche Störungsdauer bei 16 Jahren (Range 3-38), bei Wiedemann bei 9 Jahren (Range 2-36), bei Sarasalo et al. [1997] in 80\% der Fälle bei mehr als 10 Jahren (Range 5 bis >40); 87\% waren verhaftet, $5 \%$ im Gefängnis. Bei McElroy et al. betrug die mittlere Stehlhäufigkeit 27 Episoden/Monat (Range 0,3-120) mit strafrechtlichen Folgen in Form von Verhaftungen (Range 1-12) und Gefängnis; bei Wiedemann betrug sie 25 Episoden/Monat (Range 0,5-90) mit Verhaftungen (Range 1-7) und Gefängnis. Sarasalo et al. weisen auf den geringen Wert der gestohlenen Objekte hin. Die überwiegend in Kaufhäusern und Einzelhandelsgeschäften gestohlenen Gegenstände wurden gehortet, weggeworfen, zurück- oder weggegeben, benutzt oder verbraucht (in 18 von 20 Fällen [McElroy et al., 1991b] bzw. in 7 von 12 Fällen [Wiedemann, 1998]).

\section{Psychopathologie, Komorbidität, familiäres Verteilungsmuster,} Funktionalität

McElroy et al. [1991a] berichten im Rahmen ihrer Literaturrecherche, die 56 psychiatrische Patienten mit zwanghaftem oder impulsivem Stehlen einschloss, dass 32 (57\%) affektive Symptome zeigten; 20 (36\%) Patienten hätten wahrscheinlich, 12 Patienten (21\%) möglicherweise die diagnostischen Kriterien für eine Major Depression oder eine bipolare Störung erfüllt. 11 Patienten (20\%) wiesen Angst, Nervosität oder Spannung auf. 19 Patienten (34\%) hatten Phobien und/oder Panikattacken und zwanghafte Symptome. 17 Patienten (30\%) lieBen zusätzlich zum Stehlen zwanghaftes Verhalten erkennen, z.B. zwanghaftes Reinigen, Händewaschen, Kontrollieren, Horten, Sammeln, Kaufen. Essstörungen, die sich vom Verlangen nach Süßigkeiten über «Fressattacken» und selbstin- duziertes Erbrechen erstreckten und wahrscheinlich die diagnostischen Kriterien für eine Bulimia nervosa erfüllten, fanden sich bei 6 (11\%) der weiblichen Fälle. Sexuelle Dysfunktionen wie Frigidität, Vaginismus, Promiskuität und sexuelle Gefühle während des Diebstahls wurden in 7 Fällen (13\%) beschrieben. Antisoziale Verhaltensweisen (z.B. Lügen, Betrug) fanden sich bei 10 Patienten (18\%).

Nach Sarasalo et al. [1996] lag bei 81\% der 37 Betroffenen mit Kleptomanie eine gegenwärtige psychische Störung vor, bei einem Drittel mit langer Vorgeschichte. Es dominierten depressive Störungen, Angst- und Schlafstörungen; bei rund einem Drittel fanden sich in der Vorgeschichte Suizidversuche.

McElroy et al. [1991b], Wiedemann [1998] und Presta et al. [2002] untersuchten Patienten mit Kleptomanie (Fallgruppen 12-20) mit strukturierten oder standardisierten Interviews. Alle Patienten wiesen wenigstens eine weitere psychische Störung auf. Unter Zugrundelegung der DSM-Diagnostik ergibt sich folgende Lebenszeit-Komorbidität (je nach Studie):

- Affektive Störungen: 15-40\% Major Depression; 0-60\% bipolare Störung (Typ I und II);

- Angststörungen: 0-60\% Zwangsstörungen; 0-40\% Panikstörung mit oder ohne Agoraphobie; 5-40\% soziale Phobie; 8-30\% einfache Phobie;

- Störungen durch psychotrope Substanzen: 33-45\% Alkoholmissbrauch/-abhängigkeit; 20-33\% Psychopharmakamissbrauch; $20-25 \%$ andere;

- Essstörungen: 42-60\% Bulimia nervosa; 10-30\% Anorexia nervosa;

- Andere Impulskontrollstörungen: 10-20\% intermittierende explosible Störung; 10-20\% pathologisches Spielen; 5-15\% Pyromanie; $15-20 \%$ Trichotillomanie;

- Sexuelle Störungen: 83\% sexuelle Dysfunktionen [Wiedemann, 1998]; 15\% sexuelle Kompulsionen [Presta et al., 2002];

- Andere: 10\% selbstverletzendes Verhalten; 25\% körperdysmorphe Störung; 15\% Aufmerksamkeitsdefizit-/Hyperaktivitätsstörung; $15 \%$ Tic-Störung.

Bei McElroy et al. [1991b] fand sich mindestens ein Suizidversuch in 6 Fällen, ferner zwanghaftes Kaufen, Horten, Kratzen sowie Symptome und Phantasien in Richtung Paraphilie (Pädophilie, sexueller Sadismus). In 60\% der Fälle gingen affektive Störungen der Kleptomanie um wenigstens 1 Jahr voraus. In $15 \%$ traten beide Störungen im gleichen Jahr auf, in $25 \%$ der Fälle kamen die affektiven Störungen nach der Kleptomanie.

Bei Verwandten ersten Grades fanden sich nach McElroy et al. vor allem Major Depression (17\%), Zwangsstörung (7\%), Alkoholmissbrauch (14\%), Bulimia nervosa (2\%) und Kleptomanie (2\%). Bei Presta et al. fanden sich bei Verwandten ersten Grades unipolare affektive Störungen (35\%), bipolare affektive Störungen (50\%) (Typ I und II), Zwangsstörungen 25\%, Alkoholmissbrauch (15\%), Panikstörung und Anorexia nervosa (je 10\%) und Kleptomanie (5\%). 
Wiedemann [1998] fand bei der Hälfte der 12 Untersuchten eine Komorbidität zu Achse-II-Störungen: narzisstische Persönlichkeitsstörung (4); histrionische und vermeidend-selbstunsichere Persönlichkeitsstörung (je 1). Wiedemann fand außerdem, dass dem kleptomanischen Stehlen regelmäßig depressive Stimmungen, eheliche und partnerschaftliche Auseinandersetzungen und Probleme, sexuelle Frustration, Konflikte mit Kollegen, Alkoholmissbrauch durch Familienangehörige und/oder Migräneattacken oder Einsamkeit vorausgingen. Aggressive, den Stehlakt begleitende Gedanken fand er in 4 Fällen; Spannungsleichterung oder Genugtuung/Befriedigung bei allen 12 und Schuldgefühle bei 8 Patienten. Einige Patienten empfanden das Stehlen als aufregend bis stimulierend in einem unterhaltsamen bis sexuellen Sinn. Einige Patienten brachten Gestohlenes zurück. Alle Patienten erlebten sich als hilflos, ihre Aktionen als sinnlos, letztlich selbstzerstörerisch und insgesamt als ich-dyston.

Nach McElroy et al. [1991b] berichteten alle Patienten von einem unwiderstehlichen Drang zu stehlen oder Angstreduktion oder Spannungserleichterung während oder kurz nach dem Stehlen. Viele empfanden die Ausführung der Handlung als sinnlos, sich aufdrängend und ich-dyston; einige beschrieben angenehme, erfreuliche Gefühle («a rush», «a thrill», «high», «euphoric», «manic»). Alle Patienten erachteten das Stehlverhalten als falsch. Die meisten gaben Schuld oder Reue an, einige versuchten Wiedergutmachungen. Ähnlich wie bei Wiedemann gaben auch bei McElroy et al. viele der Untersuchten eine Verbindung zwischen affektiven und kleptomanischen Symptomen an, z.B. einen Wechsel in der Stehlhäufigkeit, wenn sie sich depressiv oder euphorisch fühlten, sowie eine Erleichterung von affektiven Symptomen durch das Stehlen.

Kleptomanes Risikosuchverhalten kann eine antidepressive Wirkung haben [Janet, 1911; Fishbain, 1987]. Goldman [1991] fand in einigen Fallberichten frühe Traumatisierungen; ein Aspekt, der seiner Meinung nach systematisch erhoben werden sollte, da Kleptomanie in solchen Fällen ein selbstschädigendes Verhalten zur Regulierung überwältigender Affektzustände sein könnte. Gudjonsson [1988] verweist in einer Fallstudie auf die Bedeutung eines niedrigen Selbstwertgefühls und allgemeiner Lebensunzufriedenheit für zwanghaften Ladendiebstahl. Diese Faktoren bildeten den Ausgang für eine Entwicklung, in der Angstgefühle, Frustration und ein Mangel an Selbstverwirklichung vorübergehend durch Ladendiebstahl erleichtert werden.

\section{Neurobiologische Befunde}

Vereinzelt wurde über das Auftreten von Kleptomanie bei Patienten nach Frontalhirnstörung [Khan und Martin, 1977; Gößling und Rosin, 1994; Kozian, 2001] und nach Läsionen der orbitofrontalen-subkortikalen Schaltkreise [Nyffeler und Regard, 2001] berichtet. Nach Kozian [2001] ist der genaue pathogenetische Zusammenhang zwischen Kleptomanie und einer Frontalhirnstörung jedoch nicht bekannt; ein Ausblei- ben der frontalen Hemmung parietal generierter Handlungsimpulse könnte in einem pathogenetischen Zusammenhang mit Kleptomanie stehen. Zudem würden biologische Befunde von Marazziti et al. [1999] und die positive therapeutische Beeinflussung von Kleptomaniepatienten mit SSRI nahelegen, dass eine Störung der Serotoninübertragung einen pathogenetischen Faktor darstellen könnte.

\section{Behandlungsansätze}

Mehrere Therapieansätze zur Behandlung der Kleptomanie wurden publiziert. Veröffentlichungen dazu umfassen einzelne oder wenige Fälle (1-20). Kontrollierte Studien liegen nicht vor.

\section{Psychodynamische Therapie}

Psychoanalytische Autoren haben Kleptomanie unter verschiedenen ätiologischen Perspektiven am häufigsten behandelt [vgl. Winer und Pollock, 1988; Goldman, 1991]. Neben konfliktorientierter wird eine Ich-stützende Therapie für notwendig erachtet [Cierpka, 1986]. Verlässliche Aussagen über die langfristige Wirksamkeit psychoanalytischer Therapie sind bislang nicht möglich [Fiedler und Mundt, 1997], da die Autoren sich vorrangig mit der Erklärung und Deutung der Kleptomanie beschäftigten.

\section{Psychopharmakotherapie}

Verschiedene Antidepressiva (vor allem SSRI), Lithium wie auch Elektrokrampftherapie wurden eingesetzt [ausführlich bei Durst et al., 2001]. Den Autoren zufolge wurde in 19 von 30 Fällen eine erfolgreiche Behandlung durch SSRIs berichtet. Nach Kozian [2001] kam es unter einer kombinierten Therapie mit Paroxetin und einer verhaltenstherapeutischen Maßnahme (nicht benannt) zu einer Abnahme der Häufigkeit der Stehlhandlungen.

\section{Verhaltenstherapie}

Mit Ausnahme von Wiedemann [1998] legten Verhaltenstherapeuten bisher nur Einzelfallstudien vor, bei denen verschiedene Therapietechniken angewendet wurden, wie Assertiveness-Training [Wolpe, 1958], Aversionstechniken, unter anderem verdeckte Sensibilisierung [Cautela, 1967; Guidry, 1975; Keutzer, 1972; Warmann, 1980; Gauthier und Pellerin, 1982; Glover, 1985]. Marzagao [1972] arbeitete mit systematischer Desensibilisierung; McConaghy und Blaszczynski [1988] arbeiteten mit imaginativer Desensibilisierung. Trainiert wurde beispielsweise, bei Aufkommen von Stehlimpulsen den Atem anzuhalten und das Kaufhaus zu verlassen [Keutzer, 1972]; oder es wurden aversive Phantasien eingeübt, die die Betroffenen beim Drang zu stehlen einsetzten [Gauthier und Pellerin, 1982; Glover, 1985]. Die erzielte Selbstkontrolle hielt in den meisten Fällen in den Nachuntersuchungszeiträumen an (10 Wochen bis 2 Jahre). 
Robertson und Meyer [1976] entwickelten für eine Patientin nach funktionaler Analyse ein Behandlungsprogramm mit therapeutischen Maßnahmen und Interventionen auf drei Gebieten: 1. Unterstützung bei der Suche nach einer Arbeitsstelle, die eine Herausforderung darstellte und ein Gefühl von Leistung vermittelte; 2. Paartherapie, um die Ehe wieder aufregender zu gestalten, vor allem auf sexuellem Gebiet; 3. wurde die Patientin angewiesen, Versuchungssituationen weiter aufzusuchen; vertraglich wurde vereinbart, dass sie jeden gestohlenen Gegenstand zurückzubringen habe, um auf diese Weise die Gefühle von Aufregung und Leistung zu empfinden, die normalerweise das Stehlverhalten verstärkten; ferner erfolgte die Einübung der für die Patientin aversiven Vorstellung, ihre Kinder könnten ihr Fehlverhalten herausfinden. Während der 6-monatigen Verhaltenstherapie und der 1-jährigen Nachbehandlung kam es zu vergleichsweise wenigen Vorfällen, die durch schwierige häusliche Situationen und psychosoziale Belastungen ausgelöst waren. Insgesamt hatte die $\mathrm{Pa}-$ tientin das zwanghafte Stehlen zum ersten Mal seit ihrer Kindheit unter Kontrolle; bisherige langjährige Vorbehandlungen hatten zu keiner Verbesserung geführt.

Kossak [1985] berichtet von einer Patientin, die durch eine Kombination von Verhaltenstherapie und Hypnose lernte, Alternativreaktionen zum Stehlen zu erkennen und einzuüben. Während der 10-monatigen Beobachtungszeit wurde von keiner kritischen Verhaltensweise mehr berichtet.

Gudjonsson [1987] beschreibt die 5 Monate dauernde, wöchentliche Behandlung einer depressiven Frau mit Handlungszwängen und zwanghaftem Stehlen (bis zu 1- bis 2$\mathrm{mal} / \mathrm{Tag}$, seit 20 Jahren und verschiedentlich vorbehandelt). Vor dem Hintergrund einer Funktionsanalyse wurde die tägliche Hausarbeit von 12 auf 2-3 Stunden reduziert und Alternativverhalten aufgebaut, das der Patientin jene Herausforderung und Selbstverwirklichung brachte, die sie sich zuvor durch Stehlen geholt hatte. Dadurch ließ der Drang zu stehlen, der vor und während ihrer Menstruation und bei stressreichen Lebensereignissen ausgeprägt war, allmählich nach, und die Patientin war in der Lage, allein und ohne Belastung einkaufen zu gehen (2-Jahres-Follow-up).

Eine kognitive Verhaltenstherapie bei zwanghaftem Stehlen illustrieren Schwartz und Hoellen [1991]. Nach 39 Sitzungen innerhalb von 16 Monaten berichtete die Behandelte von einem befriedigenden Sexualleben und einer deutlich besseren Beziehung zu ihrem Ehemann. Sie konnte in Supermärkte gehen, «ohne groß unter ihrem Zwang zu leiden, d.h. ohne etwas einzustecken» [ebd., S. 24]. In den Follow-up-Sitzungen 3 und 5 Jahre später gab die Behandelte an, dass die Behandlungsfortschritte ohne Ausnahme anhielten.

Wiedemann [1998] berichtet über eine von 1981 bis 1984 in der Psychosomatischen Klinik in Windach durchgeführte Studie. 12 Patienten mit Kleptomanie wurden mit einer multimodalen Verhaltenstherapie behandelt. Diese beinhaltete nach einer individuellen Verhaltensanalyse und Behandlungsplanung Therapieelemente wie Kommunikationstraining (7 Pa- tienten), Training sozialer Fertigkeiten (6 Patienten), Entspannungstraining nach Jacobson (4 Patienten), Rollenspiele (4 Patienten), Körperwahrnehmungstraining (2 Patienten), Konfrontationstraining (in sensu und in vivo; 5 Patienten), Essstörungsgruppe (3 Patienten), Ehetherapie (6 Patienten) oder Familientherapie (1 Patient). Die Mehrzahl der Patienten erhielt außerdem ein Expositionstraining (in sensu und in vivo) bezüglich des kleptomanischen Verhaltens und der Impulse (nicht näher ausgeführt). Bei Therapieende zeigten 6 Patienten keine kleptomanischen Impulse mehr, 4 Patienten hatten sich insgesamt deutlich verbessert, 2 waren unverändert. 8 Patienten konnten nach 5 Monaten bis 3,2 Jahren nachuntersucht werden; die Behandlungsergebnisse blieben stabil.

\section{Einleitung zur Untersuchung}

Das der Studie zugrunde liegende Verständnis- und Interventionsmodell beruht auf Modellbildungen aus der Hamburger Verhaltenstherapie-Ambulanz zu Zwangs- und Zwangsspektrumsstörungen [ausführlich bei Hand, 1989, 1992, 1997, 1998; Hand und Kaunisto, 1984]. In Anlehnung an das Modell zur Behandlung pathologischer Spieler beinhaltete der Therapieansatz die Beibehaltung eigener Verhaltensfreiräume und Entscheidungsfreiheiten für den Patienten und damit auch die Eigenverantwortlichkeit für das Stehlverhalten. «Stehlabstinenz» oder kontrolliertes Stehlen waren nicht Therapieziele und wurden von den Teilnehmern nicht gefordert. Die Interventionen erfolgten auf der Ebene der «Ursachen» - im Sinne der herausgearbeiteten auslösenden und aufrechterhaltenden Bedingungen und Funktionen - für das kleptomanische Verhalten.

\section{Patienten und Methode}

\section{Stichprobe}

Um eine Stichprobe zu gewinnen, wurde eine Meldung an alle Hamburger Tageszeitungen gegeben, die auf die Studie aufmerksam machte. Es meldeten sich 22 Betroffene. Sie waren von behandelnden Fachärzten ( $\mathrm{n}=13)$, Bewährungshelfern oder Rechtsanwälten $(\mathrm{n}=4)$ oder Lebenspartnern $(\mathrm{n}=5)$ auf die Behandlungsmöglichkeit bei dieser Störungsform hingewiesen worden. Um die Stichprobenpopulation überregional vergleichbar zu operationalisieren, sollten die Kriterien des DSM-III-R [APA, 1987] erfüllt sein. Ferner war die Bereitschaft zur Teilnahme an der Studie, einschließlich der «Ursachentherapie», Voraussetzung. An der Untersuchung beteiligten sich 16 Patienten. Ausgewertet wurden die Daten aller Teilnehmer, soweit sie vorlagen. Dargestellt werden die Angaben von 12 Teilnehmern, die die Untersuchung und die Behandlung (einschließlich Follow-up) regulär beendeten und von denen alle Daten vorhanden waren.

Tabelle 1 gibt einen Überblick über den Sozialstatus der Patienten und über klinisch relevante Merkmale. Die Anzahl rechtskräftiger Verurteilungen wegen «Diebstahls geringwertiger Sachen» variierte in der Stichprobe zwischen 2 und 13 (in 2 Fällen Gefängnis). Eine Therapieauflage lag aktuell in keinem Fall vor. 8 Patienten waren ambulant oder stationär wegen Depression und Angst, Adipositas, Migräne, Psoriasis oder Alkoholmissbrauch vorbehandelt, bei 6 Patienten bestand eine enge ärztliche 
Tab. 1. Soziodemographische und klinische Merkmale der Stichprobe $(\mathrm{n}=12)$

\begin{tabular}{|c|c|c|}
\hline & $\mathrm{n}$ & $\%$ \\
\hline \multicolumn{3}{|l|}{ Geschlecht } \\
\hline - Männlich & 11 & 92 \\
\hline - Weiblich & 1 & 8 \\
\hline \multicolumn{3}{|l|}{ Partnerschaft } \\
\hline - Ehe/Partnerschaft & 10 & 83 \\
\hline - Keine Ehe/Partnerschaft & 2 & 17 \\
\hline \multicolumn{3}{|l|}{ Schulabschluss } \\
\hline - Hauptschule & 7 & 58 \\
\hline - Mittlere Reife & 3 & 25 \\
\hline - Abitur & 2 & 17 \\
\hline Zusatzausbildung (Lehre/Studium) & 8 & 67 \\
\hline Berufstätig & 4 & 33 \\
\hline \multicolumn{3}{|l|}{ Nicht berufstätig (Hausfrau/Rente/ } \\
\hline \multirow{2}{*}{ Umschulung/Studium/arbeitslos) } & 8 & 67 \\
\hline & $\mathrm{M}(\mathrm{SD})$ & Range \\
\hline Alter, Jahre & $44,8(15,2)$ & $25-70$ \\
\hline Erstmanifestationsalter, Jahre & $30,5(18,9)$ & $5-67$ \\
\hline Störungsdauer, Jahre & $14,3(12,4)$ & $1-46$ \\
\hline Jährliche Stehlhäufigkeit, Vorfälle & $54,7(50,5)$ & $2-150$ \\
\hline
\end{tabular}

Anbindung wegen verschiedener körperlicher Erkrankungen und Beschwerden. 2 Patienten hatten in der Vergangenheit wegen der Stehlproblematik Hilfe in einer Gesprächspsychotherapie, 1 Patient in einer Verhaltenstherapie gesucht, 1 Patientin kontaktierte eine gemeindedienstliche Beratungsstelle, 1 andere einen Gemeindepfarrer, 3 konsultierten einen Psychiater. Die aktuelle Vormedikation bestand bei 4 Patienten aus einem Antidepressivum (bei Therapieende und Abschluss der Studie: je 2 Patienten).

\section{Untersuchungsablauf, -verfahren}

Nach Aufklärung über die Studie und Einverständniserklärung erfolgte ein psychiatrisches Erstgespräch zur Indikationsstellung. Beim zweiten Untersuchungstermin wurde das CIDI [Composite International Diagnostic Interview, dt. Bearbeitung Semler, 1987] im Bereich affektive Störungen durchgeführt. Der dritte Untersuchungsabschnitt vor Beginn der Behandlung beinhaltete die biographische Anamnese sowie eine funktionale Verhaltensanalyse zum kleptomanischen Verhalten und anderen Konflikt- und Problembereichen. Der Studie lagen ein VerhaltensanalyseSchema und ein multisymptomatisches Testpaket aus der Hamburger Verhaltenstherapie-Ambulanz zugrunde, ergänzt durch einen Fragebogen zum Problemverhalten. Handlungs- und Gedankenzwänge wurden mit dem Hamburger Zwangsinventar [HZI-K; Klepsch et al., 1993], emotionale Beeinträchtigungen ängstlich-depressiver Qualität durch die Depressionsskala [D-S; von Zerssen, 1976], phobische und soziale Ängste durch das Fear Survey Schedule [FSS-S, FSS-P; Hallam und Hafner, 1978] und Persönlichkeitsmerkmale mittels des Freiburger Persönlichkeitsinventars [FPI-R; Fahrenberg et al., 1984] erhoben. Den Fragebogen zum Problemverhalten entwickelte die Verfasserin in Anlehnung an einschlägige Literatur. Er deckt in Form von halboffenen, offenen und kategorisierten Fragen folgende Gebiete ab: Soziale Situation; Vorgeschichte und Entwicklung des kleptomanischen Verhaltens; Gestohlenes; Verwendung; Geschädigte; strafrechtliche Folgen; Merkmale der Ebene des Verhaltens und Erlebens wie der generellen Befindlichkeit vor, während und nach der Stehlhandlung; Organismusvariablen. Ferner gaben die Patienten in
Tab. 2. Merkmale kleptomanischen Verhaltens in der Stichprobe

«Nur» Kaufhäuser, Geschäfte mit Selbstbedienung

Verschiedene Artikel, identische Exemplare

Verschenken, achtlos liegen lassen, wegwerfen, zurückgeben, horten

Keine Organisation, impulsiv-dranghaft, zwangsartig, immer auf dieselbe Art und Weise

Vor der Handlung: steigende subjektive Spannung oder subjektives Unbehagen

Während oder sofort nach der Handlung: Entspannung, Erleichterung,

Stimulation, Befriedigung, Genugtuung

Schuldgefühle, Selbstvorwürfe

Bewertung als unvernünftig; Hilflosigkeit

Gewählte Selbstkontrollmaßnahmen bleiben langfristig unwirksam

diesem Fragebogen a) die Behinderung durch das kleptomanische Verhalten in 8 verschiedenen (Lebens-)Bereichen an (Partnerschaft, Familie, Berufs-/Haushaltstätigkeit/Ausbildung, soziale Kontakte, Freizeitverhalten, Selbstsicherheit/-vertrauen, psychisches und körperliches Befinden) sowie b) Stehlintensität und Belastung durch das Stehlen. Die Einschätzung erfolgte anhand von visuellen Analogskalen $(0=$ keine Behinderung bzw. kein Stehlen bzw. keine Belastung bis $8=$ extreme Behinderung bzw. exzessives Stehlen bzw. extreme Belastung). Zusätzlich wurde zum Abschluss der Studie eine Global-Rating erhoben, in dem sich die Patienten als gebessert, verschlechtert oder unverändert im Vergleich zu Untersuchungsbeginn einschätzten. Eine etwaige Symptomverschiebung wurde im Abschlussgespräch systematisch erfragt.

Die Therapieevaluation erfolgte mangels allgemein gültiger Bewertungskriterien bei Kleptomanie in Anlehnung an das «Spielerprojekt» [vgl. Klepsch et al., 1989] unter Berücksichtigung eines 35-\%-Kriteriums. Drei Erfolgskriterien-Komplexe lagen der Beurteilung zugrunde:

1. Reduktion von mindestens $35 \%$ des Mittelwerts in allen vorstehend genannten (Lebens-)Bereichen vom Untersuchungsbeginn zum Abschluss der Studie; ferner Berücksichtigung der Globaleinschätzung des Patienten.

2. Reduktion von mindestens $35 \%$ im Vergleich zum Ausgangswert in den Skalen Stehlintensität und Belastung.

3. Keine Symptomverschiebung.

Als sehr gebessert wurden Patienten beurteilt, die alle Kriterien gleichzeitig erfüllten. Als deutlich gebessert wurden Patienten beurteilt, die sich im Global-Rating als gebessert einstuften, keine Symptomverschiebung zeigten und lediglich eines der übrigen Erfolgskriterien nicht erfüllten. Als wenig gebessert wurden Patienten beurteilt, die sich als gebessert einschätzten, aber mindestens zwei der Erfolgskriterien nicht erfüllten. Als nicht gebessert wurden Patienten beurteilt, die sich als unverändert einschätzten und insgesamt mindestens zwei der Kriterien nicht erfüllten.

Als weiteres Maß für Veränderungen werden Ergebnisse aus Symptomund Persönlichkeitsskalen zu vier Messzeitpunkten im Gruppenmittel dargestellt.

Die Interviews, Behandlung, Nachuntersuchung und Auswertung führte die Verfasserin durch, begleitet von einer wöchentlichen Supervision durch ein multiprofessionelles Kernteam der Verhaltenstherapie-Ambulanz. Die mittlere Therapiedauer (diagnostische und verhaltenstherapeutische Phase zusammen) betrug 25,5 Stunden (SD 9,3; Range 12-43,5). Der mittlere Follow-up-Zeitraum bis zum Abschluss der Studie lag bei 14 Monaten (SD 4,2; Range 8-22).

Therapie

Jede Therapie war eingebettet in eine Verhaltens-, Bedingungs- und Funktionsanalyse zum kleptomanischen Verhalten sowie zu anderen Konflikt- und Problembereichen. Aus dem individuellen Störungsmodell leiteten sich die Therapieziele und der Behandlungsplan ab. Neben interak- 
tiven, bedingungsanalytischen Gesprächen beinhaltete die Behandlung je nach individueller Hintergrundproblematik ein Training kommunikativer, sozialer Fähigkeiten zur Verbesserung der Interaktions- und Problemlösefertigkeiten im Rahmen sozialer Kontakte und/oder einer problematischen Partnerbeziehung; Training der Wahrnehmung und Kommunikation von Gefühlen, persönlichen Empfindlichkeiten und Bedürfnissen, Korrektur überzogener Erwartungen; Partnergespräche; Depressionsund Angstbewältigung (biographisch und/oder aktuell) mit konkreter Hilfestellung bei Entscheidungsfindungen im privaten und beruflichen Bereich; Aufbau von Sozialkontakten und attraktivem Genussverhalten. Sicherung und Aufrechterhaltung von Veränderungen. Bei den Patienten wurde insbesondere auch auf die Erarbeitung und Etablierung alternativer Verstärker und Lösungsstrategien geachtet, da das kleptomanische Stehlen «in der Tat» Stimulation, Herausforderung, ein Gefühl von Leistung und anderes für die Stichprobenpatienten bedeutete.

\section{Ergebnisse}

Ergebnisse aus dem Fragebogen zum Problemverhalten und der funktionalen Verhaltensanalyse

Merkmale kleptomanischen Verhaltens in der Stichprobe zeigt Tabelle 2 im Überblick. Manche Patienten stahlen «nur» in Kaufhäusern, andere auch in Einzelhandelsgeschäften mit Selbstbedienung. Gestohlen wurden Süßigkeiten, Lebensmittel, Kosmetik, Kleidungsstücke, Handtaschen, Schreib-, Spiel- und Haushaltswaren, Foto- und Bastelbedarf, Nagelschere, Stickereien, eine Flasche Frauengold, Bücher, Zeitungen, Zeitschriften, Zollstöcke, Nägel, Schrauben, Birnenfassungen, Rasierklingen. Häufig wurden identische Exemplare eines Artikels immer wieder gestohlen (wie Handtaschen, Blusen, Gardinenrollen, ohne Gardinen zu besitzen oder besitzen zu wollen, Kämme, kleine Glas- und Porzellanteile; Rasierklingen (Fall 4): «Ich wollte mit meinem Leben Schluss machen, aber ich war zu feige.») Das Entwendete wurde von den Patienten (Mehrfachnennungen) verschenkt $(8 \times)$, achtlos liegen gelassen $(6 \times)$, weggeworfen $(4 \times)$, im Geschäft zurückgelegt oder anonym zurückgeschickt $(4 \times)$. Eine Patientin gab an, bei jedem Stehlen gestellt worden zu sein. Einzelnes wurde auch benutzt oder verbraucht («wenn es nun mal zu Hause war») wie z.B. Lippen- oder Augenbrauenstift oder im Wohnbereich aufgestellt (z.B. kleine Glas- und Porzellanteile) oder in einem Fall im verschlossenen Kleiderschrank gehortet, um sich im Nachhinein (beim Anblick des Gestohlenen) sexuell zu befriedigen. Abgesehen von zwei Patienten, deren Stehlverhalten im Rahmen von «Peergroup»-Kontakten begann, wurden die Delikte allein, nicht organisiert und ohne auffällige Verdeckungsversuche ausgeführt.

Die Patienten gaben an, entweder impulsiv (plötzlich, unreflektiert aus dem Moment heraus) oder aus einem Gefühl «wie getrieben», «wie angetrieben» oder aus einem «drängenden Gefühl», «Drang, es tun zu müssen», zu stehlen. Alle Patienten beschrieben steigende Spannung oder Unbehagen unmittelbar vor der Handlung sowie Entspannung, Erleichterung, Stimulation, Befriedigung oder Genugtuung; daneben «Nervenkitzel», «Freude» (3) während des Stehlens oder
Tab. 3. Auslösende und aufrechterhaltende Bedingungen und Funktionen des kleptomanischen Verhaltens

\section{Bedingungen}

Konflikte in Partnerschaft, Familie, Beruf und anderen sozialen Bezügen

Situationale oder personale Umstellungen, subjektiv belastende und überfordernde Lebenssituationen

Negative Befindlichkeiten bis zu depressiven Zuständen

Störungen des Selbstwertgefühls, unverarbeitete Kränkungen

Kurzfristige Konsequenzen:

- Entspannung, Erleichterung, Stimulation, Vergnügen, Befriedigung, Genugtuung $\mathrm{C}^{+}$

- Abgelenktsein von als aversiv empfundenen inneren und äußeren Bedingungen $\ell^{-}$

\section{Funktionen}

Intraindividuell: spannungslösende, kompensierende, stabilisierende Funktion

Interaktionell: kommunikative Funktion (Aufmerksamkeits- und Hilfsappell, Protestreaktion, Kompensation zwischenmenschlicher «Ohnmacht»)

gleich danach. Sexuelle Erregung, Orgasmus, Masturbation während des Stehlens wurde von keinem Patienten angegeben. Der Handlung folgten Schuldgefühle und Selbstvorwürfe (12), die mit Reaktionen wie Übelkeit, Schwindel, Appetitlosigkeit einhergingen (3). 5 Patienten litten unter Schuldgefühlen, besonders der Familie gegenüber, und distanzieren sich von ihrem Verhalten durch kognitive Bewältigung wie Rationalisieren, Verharmlosen (z.B. «nie jemanden persönlich bestohlen», «nur in Kaufhäusern gestohlen», «nur Kleinkram weggenommen»). Alle Patienten bewerteten ihr Verhalten als «unvernünftig», gaben an, nicht aus Gründen materiellen Gewinns zu stehlen und standen ihrem Verhalten eher hilflos gegenüber. Alle fühlten sich durch das Stehlverhalten belastet und hatten Angst vor einem erneuten Rückfall. Sie versuchten, Situationen und Reaktionen durch verschiedene Maßnahmen zu kontrollieren: vermieden Einkaufsbummel oder kauften nach Regeln ein, wie sich auf eine Einkaufsliste zu konzentrieren und das Geschäft schnell wieder zu verlassen, oder nahmen durchlöcherte Stoffbeutel zum Einkaufen mit oder erledigten Großeinkäufe mit anderen oder ließen andere einkaufen oder distanzierten sich vom Handlungsimpuls, indem sie an mögliche Konsequenzen dachten, oder sie versuchten, ihre Handlung durch Willenskraft und Selbstinstruktionen zu beeinflussen. Die gewählten Selbstkontrollmaßnahmen erwiesen sich langfristig als nicht wirksam, früher oder später kam es bei allen Patienten wieder zu Handlungsdurchbrüchen.

\section{Auslösende und aufrechterhaltende Bedingungen}

Partnern und/oder Freunden wurde das Verhalten (je nach Fall und Funktionalität) früher oder später «gebeichtet», überwiegend erst nach einer Zeit des Verschweigens und auf äußeren Druck (z.B. nach dem ersten Gestelltwerden oder nach einschlägiger Post). In einem Fall war das Stehlen nur einem Arzt bekannt. Die berichtete Reaktion der Partner war 
Tab. 4. Mittelwerte der untersuchten Symptom- und Persönlichkeitsvariablen zu 4 Messzeitpunkten $(n=12)$

\begin{tabular}{|c|c|c|c|c|c|c|c|c|}
\hline & \multicolumn{2}{|c|}{$\begin{array}{l}\text { Prä (Untersuchungs- } \\
\text { beginn) }\end{array}$} & \multicolumn{2}{|c|}{ Post (Therapieende) } & \multicolumn{2}{|c|}{$\begin{array}{l}\text { Follow-up (6 Monate } \\
\text { nach Therapie) }\end{array}$} & \multicolumn{2}{|c|}{$\begin{array}{l}\text { Follow-up (durchschn. } \\
14 \text { Monate nach Therapie) }\end{array}$} \\
\hline & M & $\mathrm{SD}$ & M & $\mathrm{SD}$ & M & $\mathrm{SD}$ & M & $\mathrm{SD}$ \\
\hline \multicolumn{9}{|l|}{ Zwänge: (HZI-K) } \\
\hline Kontrollieren & 3,75 & 2,22 & 2,42 & 1,56 & 2,42 & 1,44 & 2,58 & 1,73 \\
\hline Waschen, reinigen & 3,92 & 2,07 & 3,42 & 1,62 & 3,67 & 2,06 & 3,50 & 1,88 \\
\hline Ordnen & 5,08 & 1,78 & 3,92 & 1,62 & 4,33 & 1,72 & 4,25 & 1,42 \\
\hline Zählen, berühren, sprechen & 3,83 & 1,70 & 3,67 & 1,78 & 3,58 & 1,73 & 3,42 & 1,16 \\
\hline Gedankenimpulse & 3,33 & 2,46 & 2,42 & 1,78 & 2,33 & 2,31 & 2,17 & 2,08 \\
\hline Katastrophengedanken & 3,33 & 1,37 & 2,67 & 1,72 & 3,25 & 2,01 & 2,67 & 1,61 \\
\hline Depression: $(D-S)$ & 16,17 & 10,81 & 8,50 & 7,69 & 8,92 & 10,23 & 9,33 & 10,85 \\
\hline \multicolumn{9}{|l|}{ Phobien:(FSS-P) } \\
\hline Soziale Ängste: (FSS-S) & 5,92 & 4,70 & 6,64 & 4,63 & 5,75 & 6,89 & 5,67 & 4,98 \\
\hline \multicolumn{9}{|l|}{ FPI-R: } \\
\hline Lebenszufriedenheit & 3,42 & 1,83 & 4,42 & 1,78 & 4,75 & 2,01 & 5,00 & 2,13 \\
\hline Soziale Orientierung & 5,25 & 1,76 & 5,08 & 1,78 & 5,00 & 1,76 & 5,08 & 1,98 \\
\hline Leistungsorientierung & 5,00 & 2,09 & 5,50 & 1,88 & 5,33 & 1,83 & 5,58 & 1,83 \\
\hline Gehemmtheit & 5,17 & 1,47 & 5,08 & 1,68 & 5,08 & 1,88 & 4,58 & 1,62 \\
\hline Erregbarkeit & 6,00 & 2,59 & 5,33 & 2,53 & 5,17 & 2,82 & 5,58 & 2,81 \\
\hline Aggressivität & 4,83 & 2,48 & 4,75 & 1,91 & 4,42 & 2,35 & 4,17 & 1,95 \\
\hline Beanspruchung & 5,50 & 2,75 & 5,33 & 1,83 & 5,08 & 2,27 & 5,42 & 2,35 \\
\hline Körperliche Beschwerden & 5,33 & 1,92 & 5,25 & 1,76 & 5,08 & 1,24 & 5,00 & 1,71 \\
\hline Gesundheitssorgen & 3,92 & 1,93 & 3,75 & 2,05 & 3,58 & 1,88 & 3,83 & 1,80 \\
\hline Offenheit & 5,92 & 1,88 & 5,58 & 2,11 & 5,67 & 2,10 & 5,50 & 1,57 \\
\hline Extraversion & 4,42 & 1,78 & 5,17 & 1,70 & 4,67 & 1,72 & 5,08 & 1,83 \\
\hline
\end{tabular}

unterschiedlich. Sie reichte von anfänglichem Verzeihen bis zum Machtspiel («wenn du stiehlst, kann ich trinken» und umgekehrt). Überwiegend wurde das als sozial nicht akzeptabel betrachtete Verhalten zum Tabu zwischen den Partnern, einhergehend mit enttäuschten Erwartungen der Patienten (7x). 6 Patienten waren mit Partnergesprächen einverstanden, die anderen lehnten einen Einbezug des Partners oder anderer Bezugspersonen ab.

Aus den Angaben der Patienten ließen sich zeitnah zum kleptomanischen Delikt regelhaft chronische und aktuelle Bedingungen finden (Tab. 3).

Im zeitlichen Umfeld der ersten oder späterer Tat(en) fanden sich situationale oder personale Umstellungen und Lebenssituationen, die von den Patienten als belastend und überfordernd erlebt wurden - wie Berentung, Wohnort- und/oder Studienplatzwechsel, Auszug der Kinder, Alkoholmissbrauch des Partners, Behinderung eines Kindes und Veränderung der Partnerbeziehung, Arbeitslosigkeit - mit Schwierigkeiten in der privaten oder beruflichen (Neu-)Orientierung, Zweifeln an getroffenen privaten oder beruflichen Entscheidungen, Trennungsambivalenz in Partnerschaften oder mit dem Gefühl, vom Partner «nicht mehr so geliebt zu werden wie früher» (bei Verlustängsten), Monotonie, das Gefühl, nicht mehr gebraucht» zu werden (bei erhöhtem Leistungsbewusstsein). 2 Patienten lebten in Beziehungen mit Alkohol missbrauchenden Partnern, ohne selbst Alkohol zu trinken. 6 Patienten fühlten sich in Beziehungen sexuell frustriert. 8 Patienten wiesen zurückliegende Erfahrungen in Beziehungen mit Eltern der Kindheit und Jugend auf, die nachhallend als ungerecht und emotional vernachlässigend empfunden wurden.

Bei 10 Patienten lagen lebensgeschichtliche Belastungen vor wie der frühe Tod der Mutter $(1 \times)$, vorübergehende Trennung von Eltern $(1 \times)$, uneheliche Geburt oder keine Erinnerung an den leiblichen Vater $(3 \times)$ oder im Erwachsenenleben der Tod des Ehemannes, der Mutter oder des Vaters oder Scheidung $(5 \times) .6$ der Patienten hatten Kriege miterlebt, mit Flucht in einem Fall. Sexueller Missbrauch wurde von keinem Patienten angegeben.

\section{Ergebnisse aus dem CIDI und den Symptom- und}

Persönlichkeitsskalen (im Gruppenmittel)

Unter Zugrundelegung der DSM-Diagnostik fand sich bei Untersuchungsbeginn in 5 Fällen $(42 \%)$ die Lebenszeitdiagnose einer Major Depression. Die Ergebnisse aus Symptomund Persönlichkeitsskalen zu vier Messzeitpunkten fasst Tabelle 4 zusammen. 
Tab. 5. Therapieerfolg/-misserfolg unter Berücksichtigung von 3 Erfolgskriterien

\begin{tabular}{|c|c|c|c|c|c|}
\hline \multirow[t]{2}{*}{ Fall-Nr. } & \multicolumn{2}{|c|}{ Erfolgskriterium 1} & \multicolumn{2}{|c|}{ Erfolgskriterium 2} & \multirow{2}{*}{$\frac{\text { Erfolgskriterium } 3}{\text { Symptomverschiebung }}$} \\
\hline & $\begin{array}{l}\text { Verbess. in } \\
\text { (Lebens-) } \\
\text { Bereichen, \% }\end{array}$ & Global Rating & $\begin{array}{l}\text { Abnahme } \\
\text { Stehlintensität, } \\
\%\end{array}$ & $\begin{array}{l}\text { Abnahme } \\
\text { Belastung, } \\
\%\end{array}$ & \\
\hline 1 & 95 & besser & 100 & 100 & keine \\
\hline 2 & 95 & besser & 100 & 100 & keine \\
\hline 3 & 100 & besser & 100 & 100 & keine \\
\hline 4 & 64 & besser & 100 & 100 & keine \\
\hline 5 & 73 & besser & 100 & 63 & keine \\
\hline 6 & 100 & besser & 100 & 100 & keine \\
\hline 7 & 35 & besser & 100 & 100 & keine \\
\hline 8 & 61 & besser & 88 & 88 & keine \\
\hline 9 & 67 & besser & 88 & 86 & keine \\
\hline 10 & 15 & besser & 75 & 14 & keine \\
\hline 11 & 61 & besser & 15 & 0 & keine \\
\hline 12 & 32 & schlechter & 0 & 13 & keine \\
\hline
\end{tabular}

Zwangssymptome lagen bei Untersuchungsbeginn im definierten Normalbereich. Ein klinisch relevantes Ausmaß (HZI-KCut-off $\geq 5$ ) zeigte sich lediglich in der Skala Ordnen. Während des Untersuchungszeitraums reduzierten sich alle Zwangssymptome, insbesondere in den Bereichen Kontrollieren, Ordnen und Gedankenimpulse (Denken von Worten und Bildern). Die durchschnittlichen Depressionswerte waren mit $\mathrm{M}=16,17$ bei Untersuchungsbeginn deutlich erhöht (Normbereich 0-10). Die Patienten erlebten eine klinisch bedeutsame Reduktion während der Therapie (bei Abschluss der Therapie $\mathrm{M}=8,50$; Normbereich), bei Abschluss der Untersuchung (im Mittel 14 Monate nach Therapieende) lag der Gruppenmittelwert weiterhin im Normbereich.

Im Bereich der Gesamtphobie und der sozialen Ängste fand sich im Gruppenmittel keine relevante Ausprägung. Das Ausmaß von Sozialphobikern (Cut-off $\geq 13$ ) lag in zwei Fällen vor, verstärkt durch Psoriasis und Adipositas in je einem Fall.

In den Persönlichkeitsskalen des FPI-R lagen im Gruppenmittel außer in der Skala Lebenszufriedenheit keine von der Norm abweichenden Ausprägungen vor. Die Lebenszufriedenheit erhöhte sich während der Behandlung und lag bei Therapieende und bei Abschluss der Studie im definierten Normbereich.

\section{Ergebnisse der Einzelfallauswertung}

Tabelle 5 gibt einen Überblick über den Therapieerfolg/-misserfolg der Patienten im Vergleich Untersuchungsbeginn zu Abschluss der Studie. Anhand der dargestellten drei Erfolgskriterien ließen sich für die 12 untersuchten, behandelten und nachuntersuchten Patienten folgende Ergebnisse feststellen: 9 Patienten (75\%) erfüllten alle drei Kriterien. Sie wiesen positive Veränderungen in den zugrunde gelegten (Lebens-)Bereichen (von mindestens $35 \%$ bis zu 100\%) sowie gleichzeitig eine Abnahme der Stehlintensität (in 7 Fällen von 100\%) und eine Reduktion der Belastung (in 6 Fällen von 100\%) auf. Diese Patienten wurden als sehr gebessert eingestuft.
2 Patienten waren wenig gebessert. Bei dem einen reduzierte sich zwar die Stehlintensität, die Abnahme der Belastung durch das Stehlen und die Verbesserung in (Lebens-)Bereichen lagen jedoch unter dem 35-\%-Kriterium. Bei dem anderen kam es zwar zu einer deutlichen Verbesserung in den (Lebens-)Bereichen, Stehlintensität und Abnahme der Belastung entsprachen jedoch nicht den geforderten Kriterien. Im Global-Rating schätzten sich beide Patienten als gebessert ein.

1 Patientin schätzte sich als verschlechtert ein. Sie gab keine Symptomverschiebung an. In allen anderen Kriterien erfüllte sie die Definition nicht.

Bezogen auf die untersuchte Stichprobe ergibt sich somit eine Erfolgsquote von $75 \%(n=9)$; bezogen auf die Eingangsstichprobe $(\mathrm{n}=16)$ liegt die Erfolgsquote bei 56,3\%. 4 Teilnehmer der Eingangsstichprobe beendeten die Diagnostikphase (1) bzw. die Therapiephase (3) nicht und konnten nicht nachbefragt werden (2 von ihnen antworteten auf das schriftliche Nachbefragungsgesuch nicht; 2 erklärten aufgrund von Examensbelastungen bzw. weil der Ehemann keinen weiteren Kontakt wünsche, nicht teilnehmen zu wollen).

\section{Schlussfolgerungen}

Kleptomanie ist eine bisher nur an kleinen Fallzahlen untersuchte Störung, systematische Untersuchungen gibt es kaum. Die Klassifikation und die klinische Beurteilung sind problematisch. Kleptomanie weist nach bisherigem Kenntnisstand Komorbidität zu anderen psychischen Störungen und psychosozialen Belastungen auf und scheint bei Frauen viel häufiger aufzutreten als bei Männern. Es besteht Forschungs- und mit einer möglichen Prävalenz von $0,6 \%$ auch Versorgungsbedarf bei Kleptomanie. Die ersten Ergebnisse der vorliegenden Anwendungs-Beobachtungsstudie sind ermutigend. Replikationsuntersuchungen mit größerer Fallzahl sind erforderlich, um die Ergebnisse zu unterstützen oder zu verwerfen. 


\section{Literatur}

Aggernaes M: A study of kleptomania with illustrative cases. Acta Psych Neurol Scand 1961;36:1-46. American Psychiatric Association (ed): Diagnostic and Statistical Manual of Mental Disorders, ed 3. Washington, DC, American Psychiatric Association, 1980.

American Psychiatric Association (ed): Diagnostic and Statistical Manual of Mental Disorders, ed 3, revised. Washington, DC, American Psychiatric Association, 1987.

American Psychiatric Association (ed): Diagnostic and Statistical Manual of Mental Disorders, ed 4. Washington, DC, American Psychiatric Association, 1994.

American Psychiatric Association (ed): Diagnostic and Statistical Manual of Mental Disorders, ed 4, Text Revision. Washington, DC, American Psychiatric Association, 2000.

Bernhard W: Die Geisteskrankheiten in Beziehung zur Medizin und Staatsarzneikunde vollständig dargestellt von E. Esquirol. Bd 2, Kap. XI. Berlin, Verlag der Voss'schen Buchhandlung, 1838, pp 1-15.

Cautela JR: Covert sensitization. Psychol Rep 1967;20: 459-468.

Cierpka M: Zur Psychodynamik der neurotisch bedingten Kleptomanie. Psychiatr Prax 1986;13:94-103.

Deutsches Institut für Medizinische Dokumentation und Information, DIMDI (Hrsg): Internationale statistische Klassifikation der Krankheiten und verwandter Gesundheitsprobleme. ICD-10-GM 2004. Köln, Deutscher Ärzte-Verlag, 2004.

Dilling H, Mombour W, Schmidt MH (Hrsg): Internationale Klassifikation psychischer Störungen (Kapite V). Bern, Huber, 1991.

Durst R, Katz G, Teitelbaum A, Zislin J, Dannon PN: Kleptomania: Diagnosis and Treatment Options. CNS Drugs 2001;15:185-195.

Esquirol E: Die Geisteskrankheiten in Beziehung zur Medizin und Staatsarzneikunde (dt. Übersetzung Bernhard W, Bd 2, Kap XI). Berlin, Verlag der Voss'schen Buchhandlung, 1838, pp 1-15.

Fahrenberg J, Hampel R, Selg H: Das Freiburger Persönlichkeitsinventar FPI (Handanweisung der revidierten Fassung FPI-R). Göttingen, Hogrefe, 1984

Fiedler P, Mundt C: Dissoziative Störungen, vorgetäuschte Störungen und Störungen der Impulskontrolle; in Hahlweg K, Ehlers A (Hrsg): Enzyklopädie der Psychologie, Bd 2, Psychische Störungen und ihre Behandlungen. Göttingen, Hogrefe, 1997, pp 408-411. Fishbain DA: Kleptomania as risk-taking behavior in response to depression. Am J Psychother 1987;41: 598-603.

Gauthier J, Pellerin D: Management of compulsive shoplifting through covert sensitization. J Behav Ther Exp Psychiatry 1982;13:73-75.

Glover JH: A case of kleptomania treated by covert sensitization. B J Clin Psychol 1985;24:213-214.

Gößling HW, Rosin J: Kleptomanie vor und nach einer spontanen Subarachnoidalblutung: Ein neuropsychodynamischer Fallbericht. Fortschr Neurol Psychiat 1994;62:164-168.

Goldman MJ: Kleptomania: Making sense of the nonsensical. Am J Psychiatry 1991;148:986-996.

Goldman MJ: Kleptomania: The Compulsion to Steal - What Can Be Done? Far Hills, NJ, New Horizon Press, 1998.

Gudjonsson GH: The significance of depression in the mechanism of 'compulsive' shoplifting. Med Sci Law 1987;27:171-176

Gudjonsson GH: Causes of compulsive shoplifting. Br J Hosp Med 1988;40:169.

Guidry LS: Use of a covert punishing contingency in compulsive stealing. J Behav Ther Exp Psychiatry 1975;6:169
Hallam RS, Hafner RJ: Fears of phobic patients: Factor analysis of self-reported data. Behav Res The 1978:16:1-6.

Hand I: Verhaltenstherapie und kognitive Therapie in der Psychiatrie; in Hand I, Wittchen HU (Hrsg): Verhaltenstherapie in der Medizin. Berlin, Springer, 1989, pp 17-41.

Hand I: Pathologisches Spielen und delinquentes Verhalten; in Payk TR (Hrsg): Dissozialität. Psychiatrische und forensische Aspekte. Stuttgart, Schattauer, 1992 pp 97-117.

Hand I: «Zwangs-Spektrum-Störungen» oder «Nichtstoffgebundene Abhängigkeiten»? in Mundt C, Linden M, Barnett W (Hrsg): Psychotherapie in der Psychiatrie. Wien, Springer, 1997, pp 209-219.

Hand I: Pathological gambling: A negative state model and its implications for behavioral treatments. CNS Spectrums 1998;3:58-71.

Hand I, Kaunisto E: Multimodale Verhaltenstherapie bei problematischem Verhalten in Glückspielsituationen («Spielsucht»). Suchtgefahren 1984;30:1-14.

Hirschmann J: Die Kleptomanie. Vorträge der 6. Lindauer Psychotherapiewoche 1955;125-135.

Hollander E, Wong CM: Obsessive-compulsive spectrum disorders. J Clin Psychiatry 1995;56:3-6.

Hudson JI, Pope HG: Affective spectrum disorder: Does antidepressant reponse identify a family of disorders with a common pathophysiology? Am J Psychiatry 1990;147:552-564.

Janet P: La kleptomanie et la dépression mentale. Jahrbuch für Psychiatrie 1911;97-103.

Khan K, Martin ICA: Kleptomania as presenting feature of cortical atrophy. Acta Psychiatr Scand 1977; 56:168-172.

Keutzer CS: Kleptomania: A direkt approach to treatment. Br J Med Psychol 1972;45:159-163.

Klepsch R, Hand I, Wlazlo Z, Friedrich B, Fischer M, Bodek D: Langzeiteffekte multimodaler Verhaltenstherapie bei krankhaftem Glücksspielen III. Suchtgefahren 1989;35:35-49.

Klepsch R, Zaworka W, Hand I, Lünenschloß K, Jauernig G: Das Hamburger Zwangsinventar, Kurzform HZI-K. Weinheim, Beltz, 1993.

Kossak HC: Verhaltenstherapie unter Hypnose Selbstkontrolltraining mit dem «hypnotischen Begleiter». Exp Klin Hypnose 1985;I(2);113-142.

Kozian R: Kleptomanie bei Frontalhirnläsion. Psychiatr Prax 2001;28:98-99.

Kraepelin E: Compendium der Psychiatrie. Leipzig, Verlag von Ambr. Abel, 1883

Kraepelin E: Psychiatrie: Ein Lehrbuch für Studierende und Ärzte. Bd II: Klinische Psychiatrie. Leipzig, Verlag von Johann Ambrosius Barth, 1904.

Lejoyeux M, McLoughlin M, Adès J: Epidemiology of behavioral dependence: Literature review and results of original studies. Eur Psychiatry 2000;15:129-134.

Marazziti D, Dell'Osso L, Presta S, Pfanner C, Rossi A, Masala I, Baroni S, Giannaccini G, Lucacchini A, Cassano GB: Platelet $[3 \mathrm{H}]$ paroxetine binding in patients with OCD-related disorders. Psychiatry Res 1999;89:223-228

Marc CC: De la folie, considérée dans ses rapports avec les questions médico-judiciaires. Paris, Ballière, 1840

Marks I: Behavioural (non-chemical) addictions. Br J Addict 1990;85:1389-1394.

Marzagao LR: Systematic desensitization treatment of kleptomania. J Behav Ther Exp Psychiatry 1972;3;327328.

Matthey A: Nouvelles recherches sur les maladies de l'esprit. Paris et Genève, 1816.

McConaghy N, Blaszczynski A: Imaginal desensitization. Aust N Z J Psychiatry 1988;22:78-82.
McElroy SL, Hudson JI, Pope HG, Keck PE: Kleptomania: Clinical characteristics and associated psychopathology. Psychol Med 1991a;21:93-108.

McElroy SL, Pope HG, Hudson JI, Keck PE, White KL: Kleptomania: A report of 20 cases. Am J Psychiatry 1991b;148:652-657.

McElroy SL, Keck PE, Phillips KA: Kleptomania, compulsive buying, and binge-eating disorder. J Clin Psychiatry 1995;56:14-27.

McNeilly DP, Burke WJ: Stealing lately: A case of lateonset kleptomania. Int J Geriatr Psychiatry 1998;13 116-121.

Möller HJ: Zur Psychopathologie von Stehlhandlungen ohne (wesentliche) Bereicherungstendenz. Archiv Psychiatr Nerven 1977;223:323-336.

Müllener ER: Die Entstehung des Kleptomaniebegriffes. Sudhoffs Arch Z Wissenschaftsgesch 1964;48 216-239.

Neudecker A, Hand I: Trichotillomanie. Verhaltensther Verhaltensmed 1999;20:465-478.

Nyffeler T, Regard M: Kleptomania in a patient with a right frontolimbic lesion. Neuropsychiatry Neuropsychol Behav Neurol 2001;14:73-76.

Pinel P: Philosophisch-medicinische Abhandlung über Geistesverirrungen oder Manie. Wien, C. Schaumburg und Compagnie, 1801

Presta S, Marazziti D, Dell' Osso L, Pfanner C, Pallant S, Cassano GB: Kleptomania: Clinical features and comorbidity in an Italian sample. Compr Psychiatry 2002 43:7-12.

Robertson J, Meyer V: Treatment of kleptomania. A case report. Scand J Behav Ther 1976;5:87-92.

Rösler M: Der Sammler; in Freyberger HJ, Dilling H (Hrsg): Fallbuch Psychiatrie (Kasuistiken zum Kapitel V der ICD-10). Bern, Huber, 1993.

Sarasalo E, Bergman B, Toth J: Personality traits and psychiatric and somatic morbidity among kleptomaniacs. Acta Psychiatr Scand 1996;94:358-364.

Sarasalo E, Bergman B, Toth J: Kleptomania-like behaviour and psychosocial characteristics among shoplifters. Legal Criminol Psychol 1997;2:1-10.

Schwartz D, Hoellen B: 'Forbidden fruit tastes especially sweet.' Cognitive-behavior therapy with a kleptomaniac woman - a case report. Psychother Priv Pract 1991;8:19-25.

Semler G: Composite International Diagnostic Interview (CIDI), Version 86/6. München, Max-Planck-Institut für Psychiatrie, 1987.

Tynes LL, White K, Steketee GS: Toward a new nosology of obsessive compulsive disorder. Compr Psychiatry 1990;31:465-480.

von Schumann HJ: Das Problem der Begutachtung von Kleptomanen. Kriminologische Schriftenreihe aus der Deutschen Kriminologischen Gesellschaft. Bd 43. Hamburg, Kriminalistik Verlag, 1969

von Schumann HJ: Entschuldbare Eigentumsdelikte: Begutachtungen und Heilbehandlungen. Heidelberg, Kriminalistik Verlag, 1988.

von Zerssen D: Klinische Selbstbeurteilungsskalen (KSb-S) aus dem Münchener Psychiatrischen Informations-System (PSYCHIS München). Weinheim, Beltz, 1976.

Warmann WA: The use of aversion therapy to treat kleptomania. Psychopathol Afr 1980;1:77-82.

Wiedemann G: Kleptomania: Characteristics of 12 cases. Eur Psychiatry 1998;13:67-77.

Winer JA, Pollock GH: Störungen der Impulskontrolle; in Freedman AM, Kaplan HI, Sadock, BJ, Peters UH (Hrsg): Psychiatrie in Praxis und Klinik. Bd 4 Psychosomatische Störungen. Stuttgart, Thieme, 1988 pp 166-184.

Wolpe J: Psychotherapy by Reciprocal Inhibition. Stanford, Stanford University Press, 1958. 